\title{
A Novel, Robust DSP-Based Indirect Rotor Position Estimation for Permanent Magnet AC Motors Without Rotor Saliency
}

\author{
Li Ying and Nesimi Ertugrul, Member, IEEE
}

\begin{abstract}
This paper proposes and implements a novel rotor position sensorless technique for PM ac motor drives, which allows acceleration from standstill and can operate under various practical operating conditions including transient speed changes. The technique developed here relies on the measurement of the phase voltages and currents of the motor. It uses the incremental values of flux linkage, and the back-EMF functions to estimate incremental rotor position. Using a phase-locked loop (PLL) algorithm, an internal closed-loop correction algorithm can correct rotor position estimation drift, which may be due to the motor parameter variations or measurement inaccuracies. The method is implemented in closed-loop using a digital signal processor (DSP), and details of the implementation are provided in the paper. To demonstrate accuracy, robustness and reliability of the position estimation scheme, the paper presents a number of real-time experimental results, including dynamic operating conditions.
\end{abstract}

Index Terms-DSP based motor control, permanent magnet motors, position estimation, sensorless operation.

\section{INTRODUCTION}

$\mathbf{O}$ $\mathrm{NE}$ of the most active areas of research in permanent magnet (PM) ac motor controls during recent years has been the investigation of new techniques for eliminating the rotor position sensor that is normally required for selfsynchronization.

Elimination of the shaft-mounted position sensor is a very desirable objective in many applications since such sensor is often one of the most expensive and fragile components in the entire drive system. The expected benefits of the indirect rotor position sensing technique are: elimination of electrical connections to sensors, reduction in size, insusceptibility to environmental factors, and improved reliability. In addition, when absolute rotor position sensors are considered, operation at zero and low speeds are also desirable features of an indirect rotor position detection method.

A number of indirect rotor position detection techniques have been proposed in the literature for PM ac motor drives. These techniques can broadly be classified in three groups.

The first group is based on back-EMF sensing [1]-[3]. Since the back-EMF voltages of a PM ac motor are functions of the rotor position, the most common approach to estimate the

Manuscript received October 18, 2002; revised October 22, 2002. Recommended by Associate Editor S. B. Leeb.

The authors are with the Department of Electrical and Electronics Engineering, Adelaide University, Adelaide 5005, Australia.

Digital Object Identifier 10.1109/TPEL.2003.809377 rotor position is to extract the rotor position information from the back-EMF waveform of an unexcited phase. However, the back-EMF voltages of the PM ac motors are too small to be sensed at low speeds. In addition, the method is affected by the switching transients from the other current carrying phases. Therefore, it is not suitable for PM ac motors with continuous current conduction and is not suitable for low-speed operation.

The second group is based on estimation techniques that usually utilize phase voltages and currents of the motor. By using these quantities, the rotor position of the motor can be determined using a state observer [4]-[6], or a Kalman filtering technique [7], [8], or by direct calculations [9]-[11]. However, these techniques are computationally intensive, and large rotor position detection errors may occur because of quantization and truncation errors, and measurement inaccuracies. Moreover, variation in the motor parameters due to temperature changes and magnetic saturation significantly affect the estimated rotor position data. Furthermore, the estimation performance may suffer under low-speed operation, mainly due to drift of the integration functions used in the algorithms. Therefore the problems listed above make the previous methods not suitable for the practical motor drives.

Finally, the third group of indirect rotor position detection techniques is based on the detection of the rotor saliency in PM ac motors, which occurs due to the asymmetrical rotor structure or magnetic saturation [12], [13]. In this group, the rotor position information can also be extracted from responses of an injected high frequency test signal [14], [15]. This method is not dependent upon the motor speed and hence can operate at low speeds including standstill. However, these techniques are not suitable for nonsalient PM motors, such as surface mount PM ac motors that have no rotor saliency.

The principal aims of this paper are to overcome the problems of the previous methods and to propose a robust solution that is suitable for practical PM ac motor drives.

The position estimation and the practical implementation issues of the proposed method are discussed in the subsequent sections of this paper. In this novel method, three phase flux linkage increments (hence no integration is required) are calculated using the measured three-phase voltages and currents, which is the main source of the estimation error in the previous techniques. Then the flux linkage increments and back-EMF functions of the motor are utilized to derive a single rotor position increment and the corresponding rotor position information is calculated. In the method, with the help of a phase-locked 
loop (PLL) algorithm, an internal closed-loop can correct the position drift that may be caused by the deviations of the motor parameters and/or by the measurement inaccuracies.

As will be demonstrated in the results section, this novel method can accurately estimate the rotor position over a wide range of operating speeds, and is robust with respect to parameter variations of the motor and measurement errors in the system. In addition, the proposed estimation scheme is not computationally intensive, which makes the real-time implementation of the method possible with a commercially available DSP (Analog Devices ADMC300).

In addition, while the motor drive operating in closed-loop without a conventional rotor position sensor, a number of real-time experimental results are obtained to demonstrate the robustness, the reliability and the performance of the novel position estimation scheme under various practical operating conditions [16].

\section{PRINCIPLES OF THE AlgORITHM}

The method developed here is suitable for a wide range of PM ac motors. However, a three-phase surface-mounted PM ac motor is considered in the development of the position estimation algorithm.

The surface-mounted PM ac motor has identical direct and quadrature inductances, so that the rotor saliency does not exist, and thus it is not possible to use methods which rely on rotor saliency. Under ideal conditions, however, a possible solution to obtain the rotor position data is to utilize the relationships with the three-phase flux linkages that are established by the permanent magnets. This approach is considered in this study.

\section{A. Mathematical Model of the Motor}

In order to obtain a general algorithm, the three-phase a-b-c modeling approach is used in this paper. Under ideal conditions, a three-phase star-connected PM motor can be modeled by a network consisting of a winding resistance, an equivalent winding inductance, and a back EMF source per phase, all connected in series. If it is assumed that the stator resistances of the windings are identical and the self and mutual inductances are constant, which is correct in the motor type considered (no rotor saliency), the voltage equations of the motor can be given in a matrix form as

$$
\left[\begin{array}{l}
v_{a} \\
v_{b} \\
v_{c}
\end{array}\right]=\left[\begin{array}{ccc}
\mathrm{R} & 0 & 0 \\
0 & \mathrm{R} & 0 \\
0 & 0 & \mathrm{R}
\end{array}\right]\left[\begin{array}{c}
i_{a} \\
i_{b} \\
i_{c}
\end{array}\right]+\left[\begin{array}{ccc}
\mathrm{L} & 0 & 0 \\
0 & \mathrm{~L} & 0 \\
0 & 0 & \mathrm{~L}
\end{array}\right] \frac{d}{d t}\left[\begin{array}{c}
i_{a} \\
i_{b} \\
i_{c}
\end{array}\right]+\left[\begin{array}{c}
e_{a} \\
e_{b} \\
e_{c}
\end{array}\right]
$$

Here $v_{\mathrm{a}}, v_{\mathrm{b}}$, and $v_{\mathrm{c}}$ are the phase voltages; $\mathrm{R}$ is the winding resistance; $i_{\mathrm{a}}, i_{\mathrm{b}}$, and $i_{\mathrm{c}}$ are the phase currents; $\mathrm{L}$ is the equivalent winding inductance and is equal to $L_{s}-M_{s}=L_{s l}+3 / 2 L_{s 0}$ where $L_{s}$ is the self inductance and $M_{s}$ is the mutual inductance of the winding; and $e_{\mathrm{a}}, e_{\mathrm{b}}, e_{\mathrm{c}}$ are the back-EMF voltages induced in each phase winding due to the flux linkages of PMs.

In the PM ac motor, the back EMF voltages are functions of the rotor position and the angular speed. Hence, for the motor with sinusoidal back-EMF's, the three-phase back-EMF voltages can be expressed as

$$
\left[\begin{array}{l}
e_{a} \\
e_{b} \\
e_{c}
\end{array}\right]=\left[\begin{array}{l}
\mathrm{K}_{e} \omega_{r} e_{1}\left(\theta_{e}\right) \\
\mathrm{K}_{e} \omega_{r} e_{2}\left(\theta_{e}\right) \\
\mathrm{K}_{e} \omega_{r} e_{3}\left(\theta_{e}\right)
\end{array}\right]=\left[\begin{array}{l}
\mathrm{K}_{e} \omega_{r} \sin \left(\theta_{e}\right) \\
\mathrm{K}_{e} \omega_{r} \sin \left(\theta_{e}+\frac{2 \pi}{3}\right) \\
\mathrm{K}_{e} \omega_{r} \sin \left(\theta_{e}-\frac{2 \pi}{3}\right)
\end{array}\right]
$$

where $\mathrm{K}_{\mathrm{e}}$ is the back EMF constant, $\omega_{r}$ is the rotor angular speed, $e_{1}\left(\theta_{\mathrm{e}}\right), e_{2}\left(\theta_{\mathrm{e}}\right)$ and $e_{3}\left(\theta_{\mathrm{e}}\right)$ are defined as the back EMF functions that varies with the rotor position, and $\theta_{\mathrm{e}}$ is the electrical rotor position that can be given by

$$
\theta_{e}=\mathrm{p} \int \omega_{r} d t
$$

where $\mathrm{p}$ is the number of pole pairs in the motor.

\section{B. Position Increment Estimation}

In the above voltage equations, the three-phase currents and voltages are directly measurable quantities. It can be seen that there is only one possible solution to derive the rotor position information from the measurable quantities and the ideal model of the motor.

The approach used to determine the rotor position here is based on the relationship between the rotor position and the flux linkage of the permanent magnets or the back-EMF voltages.

However, if the relationship between the rotor position and the back-EMF voltages is used, the position estimation scheme may not operate accurately at low speeds due to the small induced back-EMF voltages. Furthermore, if the relationship between the rotor position and the flux linkage of the permanent magnets is utilized to calculate the total flux linkages of the windings, some form of digital integration is needed. However, digital integration is difficult to accurately realize in the real-time systems. This is due to motor parameter variations and measurement errors such as DC offsets, quantization and truncation errors. All these errors can cause integration drift and pose a significant problem in the estimation of the flux linkages, specifically at low speeds.

To overcome the drawbacks discussed above, a novel position estimation scheme is proposed here. In the new method, the calculation of the flux linkage by digital integration is eliminated and a direct calculation of the flux linkage increment change using the voltage equations is utilized. In addition, the back-EMF voltages are disassembled as the multiplication of the angular speed and the unit back-EMF functions, so that the estimation of the back-EMF function becomes independent of the angular speed.

Substituting (2) into (1), the voltage equations can be rewritten as

$$
\begin{aligned}
{\left[\begin{array}{l}
v_{a} \\
v_{b} \\
v_{c}
\end{array}\right]=} & {\left[\begin{array}{lll}
\mathrm{R} & 0 & 0 \\
0 & \mathrm{R} & 0 \\
0 & 0 & \mathrm{R}
\end{array}\right]\left[\begin{array}{c}
i_{a} \\
i_{b} \\
i_{c}
\end{array}\right] } \\
& +\left[\begin{array}{lll}
\mathrm{L} & 0 & 0 \\
0 & \mathrm{~L} & 0 \\
0 & 0 & \mathrm{~L}
\end{array}\right] \frac{d}{d t}\left[\begin{array}{l}
i_{a} \\
i_{b} \\
i_{c}
\end{array}\right]+\frac{\mathrm{K}_{e}}{\mathrm{p}} \cdot \frac{d \theta_{e}}{d t}\left[\begin{array}{l}
e_{1}\left(\theta_{e}\right) \\
e_{2}\left(\theta_{e}\right) \\
e_{3}\left(\theta_{e}\right)
\end{array}\right]
\end{aligned}
$$

As can be seen in the above equation, if the sampling frequency is high enough in a practical measurement system, the 
rotor position increment within one sample cycle can be derived in the discrete form as

$$
\left[\begin{array}{l}
\Delta \theta_{a e} \\
\Delta \theta_{b e} \\
\Delta \theta_{c e}
\end{array}\right]=\frac{\mathrm{p}}{\mathrm{K}_{e}}\left[\begin{array}{c}
\frac{\left[\left(\mathrm{V}_{a}-\mathrm{R} i_{a}\right) \cdot \Delta \mathrm{t}-\mathrm{L} \cdot \Delta i_{a}\right]}{e_{1}\left(\theta_{e}\right)} \\
\frac{\left[\left(\mathrm{V}_{b}-\mathrm{R} i_{b}\right) \cdot \Delta \mathrm{t}-\mathrm{L} \cdot \Delta i_{b}\right]}{e_{2}\left(\theta_{e}\right)} \\
\frac{\left[\left(\mathrm{V}_{c}-\mathrm{R} i_{c}\right) \cdot \Delta \mathrm{t}-\mathrm{L} \cdot \Delta i_{c}\right]}{e_{3}\left(\theta_{e}\right)}
\end{array}\right]=\frac{\mathrm{p}}{\mathrm{K}_{e}}\left[\begin{array}{c}
\frac{\Delta \psi_{a}}{e_{1}\left(\theta_{e}\right)} \\
\frac{\Delta \psi_{b}}{e_{2}\left(\theta_{e}\right)} \\
\frac{\Delta \psi_{c}}{e_{3}\left(\theta_{e}\right)}
\end{array}\right]
$$

where $\Delta \mathrm{t}$ is the sampling interval, $\Delta i_{1}, \Delta i_{2}$ and $\Delta i_{3}$ are the current increments within the sampling interval of the threephase currents, $\Delta \Psi_{\mathrm{a}}, \Delta \Psi_{\mathrm{b}}$ and $\Delta \Psi_{\mathrm{c}}$ are defined as the flux linkage increments within the sampling interval of each phase.

Ideally, each motor phase should produce identical rotor position increment $\left(\Delta \theta=\Delta \theta_{\mathrm{ae}}=\Delta \theta_{\mathrm{be}}=\Delta \theta_{\mathrm{ce}}\right)$. In practice, however, any estimation errors could cause variations in the estimated rotor position increment. In this study, a single value of position increment is obtained by averaging the three values of the position increments estimated in (5).

$$
\Delta \theta_{e}=\frac{\Delta \theta_{a e}+\Delta \theta_{b e}+\Delta \theta_{c e}}{3} .
$$

In addition, assuming that the previous value of the rotor position estimation is known, an updated value of the rotor position can be estimated simply by

$$
\theta_{e}(\mathrm{k})=\theta_{e}(\mathrm{k}-1)+\Delta \theta_{e} \quad \mathrm{k}=1,2,3 \ldots
$$

where, $k$ is an integer representing the estimation of discrete data at the $k$ th instant of time.

\section{Position Estimation Algorithm}

As can be seen in (5), since the back EMF function of each phase becomes zero at the corresponding rotor pole, the rotor position increment estimation under this condition may produce an infinitely high value. However, the remaining two phases can provide a finite and more accurate rotor position value. This means that each phase has its own optimal sensing region, where the rotor position increment estimation is most accurate. Although (6) can be used to calculate the single value of rotor position increment, in a more practical algorithm, the three rotor position increments should be weighted or adjusted with respect to their optimal sensing regions in order to prevent the loss of rotor position information and the reduction of the accuracy.

In order to utilize the optimal sensing region of each phase fully and to improve the position estimation accuracy, (5) should be rewritten in the format given as follows:

$$
\begin{gathered}
{\left[\begin{array}{c}
\Delta \theta_{a e} e_{1}\left(\theta_{e}\right) \\
\Delta \theta_{b e} e_{2}\left(\theta_{e}\right) \\
\Delta \theta_{c e} e_{3}\left(\theta_{e}\right)
\end{array}\right]=\frac{\mathrm{p}}{\mathrm{K}_{e}}\left[\begin{array}{l}
{\left[\left(\mathrm{v}_{a}-\mathrm{R} i_{a}\right) \cdot \Delta \mathrm{t}-\mathrm{L} \cdot \Delta i_{a}\right]} \\
{\left[\left(\mathrm{v}_{b}-\mathrm{R} i_{b}\right) \cdot \Delta \mathrm{t}-\mathrm{L} \cdot \Delta i_{b}\right]} \\
{\left[\left(\mathrm{v}_{c}-\mathrm{R} i_{c}\right) \cdot \Delta \mathrm{t}-\mathrm{L} \cdot \Delta i_{c}\right]}
\end{array}\right]} \\
=\frac{\mathrm{p}}{\mathrm{K}_{e}}\left[\begin{array}{c}
\Delta \psi_{a} \\
\Delta \psi_{b} \\
\Delta \psi_{c}
\end{array}\right]
\end{gathered}
$$

In (8), if the two sides of the equations inside the matrix are multiplied by $e_{2}\left(\theta_{\mathrm{e}}\right), e_{3}\left(\theta_{\mathrm{e}}\right)$ and $e_{1}\left(\theta_{\mathrm{e}}\right)$ respectively, and the three equations are summed, a single rotor position increment can be obtained as

$$
\Delta \theta_{e}=\frac{\mathrm{p}}{\mathrm{K}_{e}} \cdot \frac{\Delta \psi_{a} e_{2}\left(\theta_{e}\right)+\Delta \psi_{b} e_{3}\left(\theta_{e}\right)+\Delta \psi_{c} e_{1}\left(\theta_{e}\right)}{e_{1}\left(\theta_{e}\right) e_{2}\left(\theta_{e}\right)+e_{2}\left(\theta_{e}\right) e_{3}\left(\theta_{e}\right)+e_{3}\left(\theta_{e}\right) e_{1}\left(\theta_{e}\right)}
$$

The above equation is the basis of the incremental rotor position estimation, which can be considered as a weighted average which takes into account the optimum regions for accurate position estimation for each phase. In a very noisy environments however, a more selective range filtering may be required.

The position estimation algorithm in this study utilizes (7)-(9). Equation (8) is used to calculate the flux linkage increments using the measured phase currents and voltages. Then, the flux linkage increments and the back EMF functions are utilized to determine the rotor position increment by (9), and the final rotor position is estimated by (7). It should be noted here that the back EMF functions are produced using their waveform functions and the estimated position signal, which form an internal closed-loop.

The algorithm has several advantages. Firstly, it is simple and not mathematically intensive, which makes it very practical to be used in DSP based control systems. Secondly, the algorithm is independent of the shape of back-EMFs, hence is suitable for both sinusoidal and trapezoidal PM ac motors (or for any other PM motors with nonideal back EMFs). Finally, the calculation of the flux linkages does not include an integration algorithm, so that integration related errors are eliminated.

Although a simple integration algorithm still exists in the position calculation (7), the internal closed-loop produced by the back-EMF function feedbacks stabilizes the position integration and corrects the position drift. This is the most important feature of the novel algorithm. In other words, the method moves the integration from the flux linkage calculations to the position calculation level and hence the problems of the integration drift are solved by the internal closed-loop correction. Furthermore, this internal correction loop improves the robustness of the rotor position estimation, which would otherwise be sensitive to parameter variations and measurement errors.

It should be noted here that further improvements were done to the position estimation algorithm to enhance the accuracy of the estimation, which is illustrated in Fig. 1. In this figure, the basic algorithm described above unchanged and two additional blocks are added to the left side of the diagram: Back-EMF Functions and Phase Difference.

\section{ANALYSIS OF ROBUSTNESS AND IMPROVEMENTS}

Under ideal conditions, the algorithm described above can produce accurate rotor position estimates. In practice, however, the parameters of the motor may vary for various reasons, such as temperature rise or magnetic saturation. In addition, there are no ideal measurement circuits in reality. Practical measurement circuits can be affected by electromagnetically noisy environment. Therefore parameter deviations and measurement inaccuracies need to be considered in real-time motor control systems as they directly affect the reliability and the robustness of the control algorithm. 


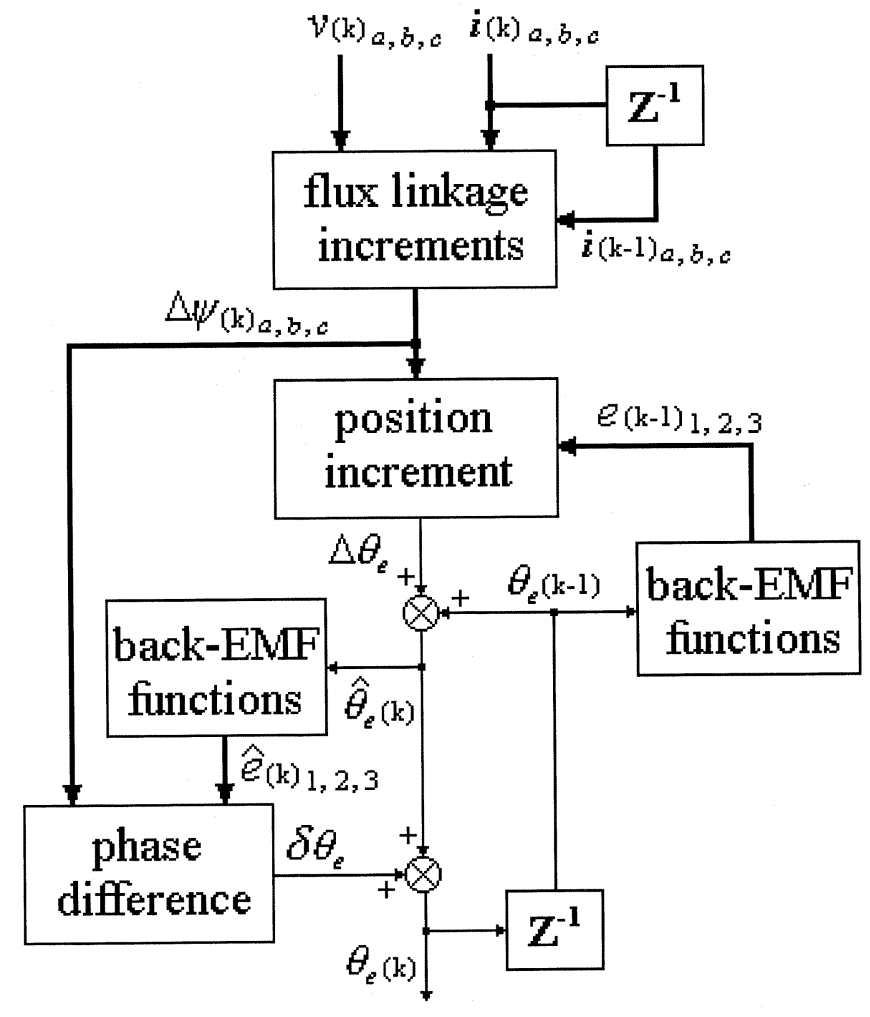

Fig. 1. Block diagram of the improved algorithm.

\section{A. Theoretical Analyzes of Robustness}

In a practical motor drive, the motor equivalent circuit parameters are nonlinear functions of time, temperature and various other parameters. Therefore, the accurate prediction of the measurement errors and noise is a challenging issue in the software/hardware integrated power electronics systems, which is difficult to predict.

It can be seen from the basic algorithm described earlier that any parameter variations or measurement inaccuracies can cause position increment errors. If no corrective action is taken, the position error may accumulate over the integration period and may cause a catastrophic failure in the drive, such as loss of synchronism.

As can be seen in (8), if the back EMF functions are ideal sinusoidal waveforms, three-phase flux linkage increments should also be ideal sinusoidal waveforms. Hence, the estimated position increment value only depends on the amplitude of the flux linkage increments. However, disturbances may occur in a nonideal (practical) system causing an error in the estimated position increment. If this error is fed back to the back-EMF functions via the internal closed-loop, it causes a phase difference between the flux linkage increments and the back-EMF functions.

Let us assume that the flux linkage increments have a phase angle of $\theta_{e}^{*}$, which reflects the actual rotor position value. Then the estimated rotor position increment based on the basic algorithm can be rewritten as

$$
\begin{aligned}
\Delta \theta_{e}= & \frac{\mathrm{p}}{\mathrm{K}_{\mathrm{e}}} \\
& \cdot \frac{\Delta \psi_{m}\left[\sin \theta_{e}^{*} \sin \left(\theta_{e}-\frac{2 \pi}{3}\right)+\sin \left(\theta_{e}^{*}-\frac{2 \pi}{3}\right) \sin \left(\theta_{e}+\frac{2 \pi}{3}\right)+\sin \left(\theta_{e}^{*}+\frac{2 \pi}{3}\right) \sin \theta_{e}\right]}{\sin \theta_{e} \sin \left(\theta_{e}-\frac{2 \pi}{3}\right)+\sin \left(\theta_{e}-\frac{2 \pi}{3}\right) \sin \left(\theta_{e}+\frac{2 \pi}{3}\right)+\sin \left(\theta_{e}+\frac{2 \pi}{3}\right) \sin \theta_{e}} \\
= & \frac{\mathrm{p} \Delta \psi_{m}}{\mathrm{~K}_{\mathrm{e}}} \cos \left(\theta_{e}^{*}-\theta_{e}-\frac{\pi}{3}\right)
\end{aligned}
$$

where, $\Delta \psi_{\mathrm{m}}$ is the amplitude of the flux linkage increment.

As can be seen from the auto-correction function given in (10), in ideal conditions, the rotor position $\theta_{e}^{*}$ is equal to the phase angle of the flux linkage increments $\left(\theta_{e}^{*}=\theta_{\mathrm{e}}\right)$, and the estimated rotor position increment is directly proportional to the amplitude of the flux linkage increments.

If an error causes the estimated rotor position to differ, the difference is fed back to the loop via the back-EMF functions and the next step value of the position increment estimation is corrected within a range of position

For example, if the estimated rotor position leads the phase angle of flux linkage increments $\left(\theta_{e}^{*}<\theta_{\mathrm{e}}\right)$, the phase difference produced by the feedback reduces the new estimated value of the position increment. Conversely, if the estimated rotor position lags the phase angle of flux linkage increments $\left(\theta_{e}^{*}>\theta_{\mathrm{e}}\right)$, the phase difference increases the new estimated value of the position increment.

\section{B. Improvements}

As discussed above, the position estimation algorithm is robust with respect to some of the measurement errors and can operate accurately within a range of position. However some static estimation errors also occur in practice, which is mainly due to the parameter variations and/or the measurement inaccuracies. The auto-correction ability is directly proportional to the motor speed. In high-speed operation, the static errors are acceptable in practical applications. At slower speeds, however, the static errors accumulate via the integration due to the poor auto-correction ability. This results in the estimated rotor position laging the flux linkage increments.

To make the position estimation algorithm resistant to static errors, a phase-locked loop (PLL) is implemented, which is proven to be very effective.

In the PLL, the three-phase flux linkage increments and the three-phase back-EMF functions are considered as vectors. By using the vector product, the phase difference between the two quantities are expressed approximately as

$$
\begin{aligned}
\|\Delta \vec{\psi} \times \vec{e}\|= & \Delta \psi_{a} e_{2}\left(\theta_{e}\right)+\Delta \psi_{b} e_{3}\left(\theta_{e}\right)+\Delta \psi_{c} e_{1}\left(\theta_{e}\right) \\
& -\Delta \psi_{a} e_{3}\left(\theta_{e}\right)-\Delta \psi_{b} e_{1}\left(\theta_{e}\right)-\Delta \psi_{c} e_{2}\left(\theta_{e}\right) \\
= & \frac{3 \sqrt{3}}{2} \psi_{m} \sin \left(\theta_{e}^{*}-\theta_{e}\right) \approx \frac{3 \sqrt{3}}{2} \psi_{m}\left(\theta_{e}^{*}-\theta_{e}\right)(11)
\end{aligned}
$$

Using the above equation in the PLL, the phase difference between the phase angle of the flux linkage increments and the estimated rotor position can be estimated. Then the value is then utilized to compensate the incremental position errors, which may be in error due to the parameter variations and/or the measurement inaccuracies.

The block diagram of the improved algorithm is given in Fig. 1, where the basic algorithm remains unchanged. If there are any parameter deviations or measurement inaccuracies in the system, however, the predicted values may be in error. Therefore, the back-EMF functions generated by the predicted rotor position will have a different phase angle from the functions generated using the flux linkage increments.

The PLL calculates the phase difference and compensates the predicted rotor position to rectify the rotor position value in the consecutive step. Therefore, the improved rotor position estimation algorithm is called the "prediction-rectification" method. 


\section{Computer Simulation Results}

To demonstrate the effectiveness of the algorithm explained above and to give some quantitative results, extensive simulation studies were performed on a sample motor, which has the parameters given in the Appendix.

The simulation studies were carried out under the steadystate conditions with a dc link voltage of $150 \mathrm{~V}$ and under the following parameter changes of the motor: $\pm 20 \% \mathrm{R}, \pm 20 \%$ $\mathrm{Ke}$ and $\pm 20 \% \mathrm{~L}$ (the winding resistance, the back-EMF constant, and the equivalent inductance errors respectively), and under the stator winding imbalances. A PWM current controller with modulation frequency of $5 \mathrm{kHz}$ is also included in the simulation.

Fig. 2 shows a set of simulation results, which aims to demonstrate the robustness with respect to the motor parameter variations.

It can be concluded from the simulation results that if the parameters of the position estimator and the motor are identical, the estimated rotor position can accurately follow the actual rotor position both in the basic and in the improved algorithms. When the parameters of the motor vary by $\pm 20 \%$, the position estimation errors do occur, but are limited to $2-3 \%$ by the basic algorithm with the help of auto-correctional action. This is an acceptable position error in a practical position sensorless motor drive, which demonstrates a degree of robustness in the basic algorithm.

However, the basic algorithm cannot eliminate static position estimation errors and the auto-correctional ability of the basic algorithm is poor at the lower speed range. By using the PLL, however, the improved algorithm significantly reduces the static estimation errors as shown in Fig. 2. It can be seen in the figure showing the position errors that the static position errors caused by the winding resistance and the back-EMF constant variations can be almost eliminated using the improved algorithm.

However, the position estimation errors caused by the equivalent inductance variations are still relatively high. This is because the variations of equivalent inductance can affect the phase of the flux linkage increments, and therefore the estimated phase of the flux linkage increments may different from the actual value if the equivalent inductance is in error.

As mentioned above, the stator winding imbalance was also studied under two different settings $\left(+10 \% \mathrm{R}_{\mathrm{a}},-10 \% \mathrm{R}_{\mathrm{c}}\right.$; and $-10 \% \mathrm{R}_{\mathrm{a}},+10 \% \mathrm{R}_{\mathrm{c}}$ ). The result indicated that there is no benefit of the improved algorithm on the stator winding imbalance. Therefore, the PLL cannot produce accurate values to rectify the errors in the rotor position estimation.

It should be emphasised here that in a practical nonsalient PM ac motor, the equivalent inductance is more stable than the other motor parameters under normal operation conditions.

Therefore, it is recommended in a practical position sensorless drive system that the equivalent inductance should be measured accurately in order to minimize the position estimation errors further.

Further simulations studies were also carried out to observe the effects of voltage and current measurement errors $( \pm 10 \%)$. In this study, the motor parameters are not changed and the similar control settings as in Fig. 2 are used. The results indicated that the basic algorithm can also limit the position errors due
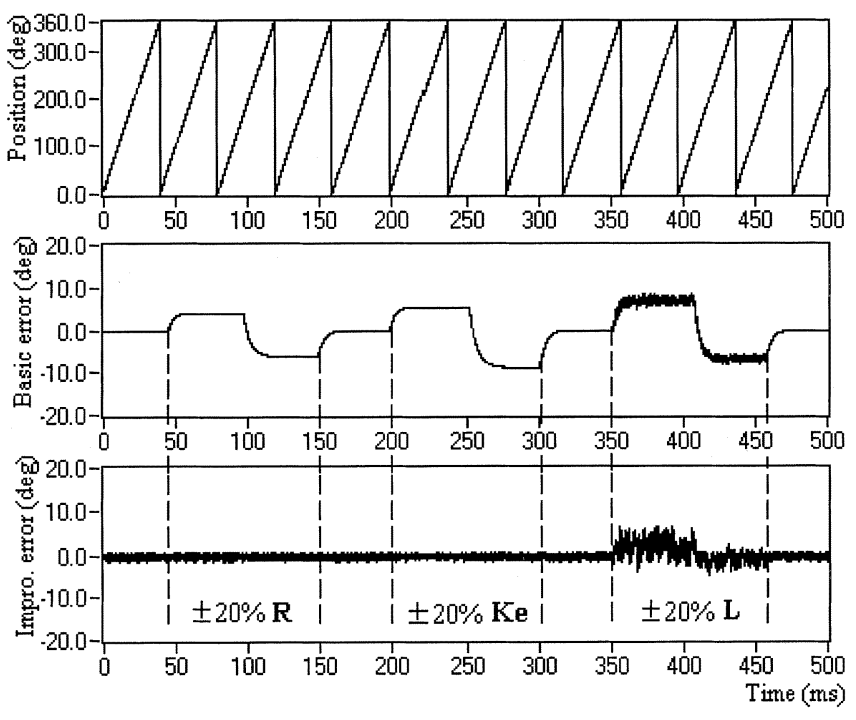

Fig. 2. Computer simulation results under the parameter changes of the motor: $\pm 20 \% \mathrm{R}, \pm 20 \% \mathrm{Ke}$ and $\pm 20 \% \mathrm{~L}$, with a command current of $2.5 \mathrm{~A}$ and at a steady-state speed of $25 \mathrm{~Hz}$. The figure shows the rotor position (top), the position errors between the actual and the estimated position signals with the basic algorithm (middle) and the position errors with the improved algorithm (bottom).

to measurement errors but the presence of the static estimation errors limit the operation at slower speeds. The improved algorithm, however, reduces the static estimation errors and extends the operating range of the motor.

\section{REAL-TIME EXPERIMENTAL RESULTS}

To test the proposed position estimation algorithm to see if it is suitable for the practical applications, a real-time experimental setup is implemented. This includes a three-phase inverter board (IRMDAC3, International Rectifiers, $460 \mathrm{~V}$, $3 \mathrm{hp}$ ) and a DSP-based motor controller from Analog Devices (ADMC 300). In addition, a PM ac motor (which has the specifications given in the appendix), a two channel incremental encoder to verify the estimated rotor position, and three high bandwidth voltage and two high bandwidth current transducers are used in the experimental setup.

\section{A. Open Loop Operation}

In the open loop test, the motor drive is synchronously controlled using the shaft mounted encoder. In the DSP, a standard PWM current controller with a PWM frequency of $5 \mathrm{KHz}$ is used to modulate the currents of the motor. In addition, a LabVIEW based data acquisition system is used to sample the real data of the motor drive at a sampling frequency of $100 \mathrm{KHz}$. Then, the measured voltage and the current data can be used to estimate the rotor position of the motor off-line and compare it with the measured rotor position.

In this section, as in the simulation studies, various degrees of artificial errors are introduced to the motor parameters of the position estimator and to the measured voltage and the current data. The performance of the algorithm has been tested under these synthetic errors.

The test results are illustrated in Fig. 3 and Fig. 4. It was observed that the position estimation algorithm can still estimate 

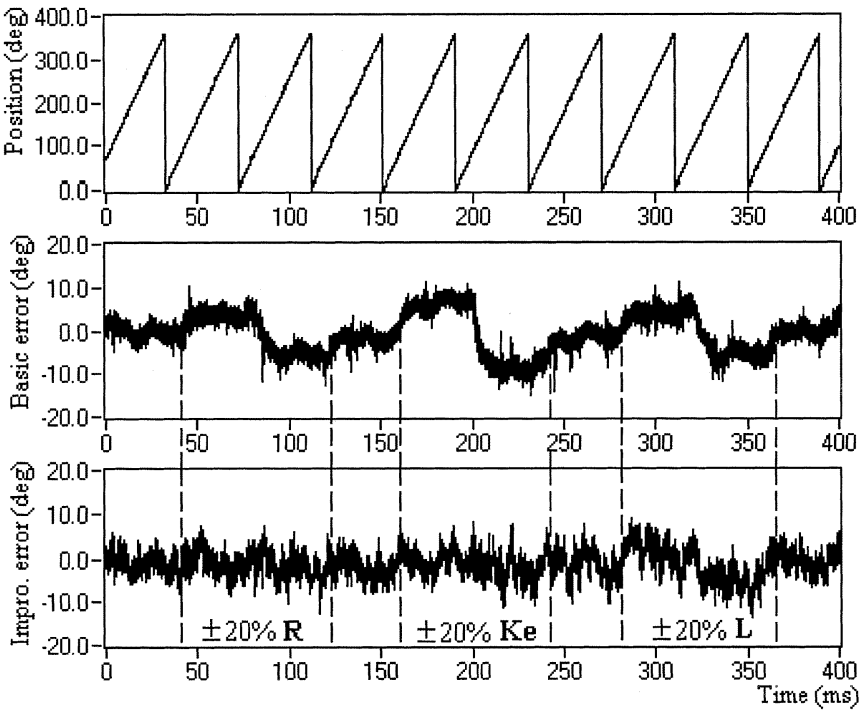

Fig. 3. Open-loop operation experimental results to demonstrate the robustness with respect to the parameter variations: the actual rotor position (top), the position estimation error using the basic algorithm (middle), and the position estimation error using the improved algorithm (bottom).
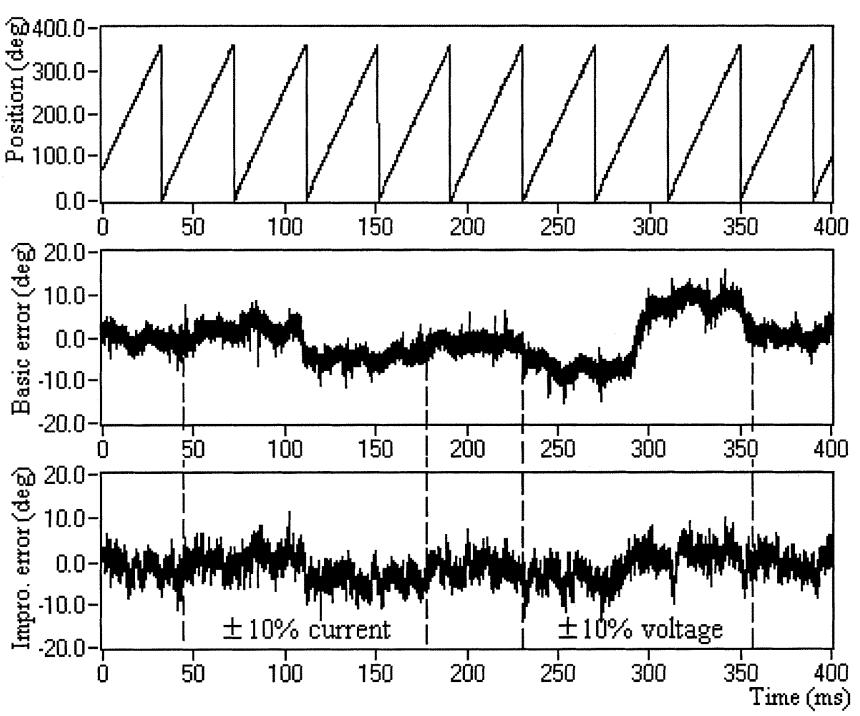

Fig. 4. Open-loop operation experimental results to demonstrate the robustness with respect to the measurement errors: the actual rotor position (top), the position estimation error using the basic algorithm (middle), and the position estimation error using the improved algorithm (bottom).

the rotor position accurately $( \pm 3-4 \%)$. Although the improved algorithm generates larger estimation noise, its static errors are smaller than the estimation errors of the basic algorithm.

\section{B. Closed-Loop Operation}

To implement position sensorless motor drive, the encoder is eliminated from the control loop, but is utilized to verify the estimated rotor position.

Fig. 5 illustrates the complete block diagram of the position sensorless motor drive system, where the position estimator replaces the shaft mounted position sensor.

Since the DSP used in this study has only five A/D converter channels, three phase voltages and only two phase currents are

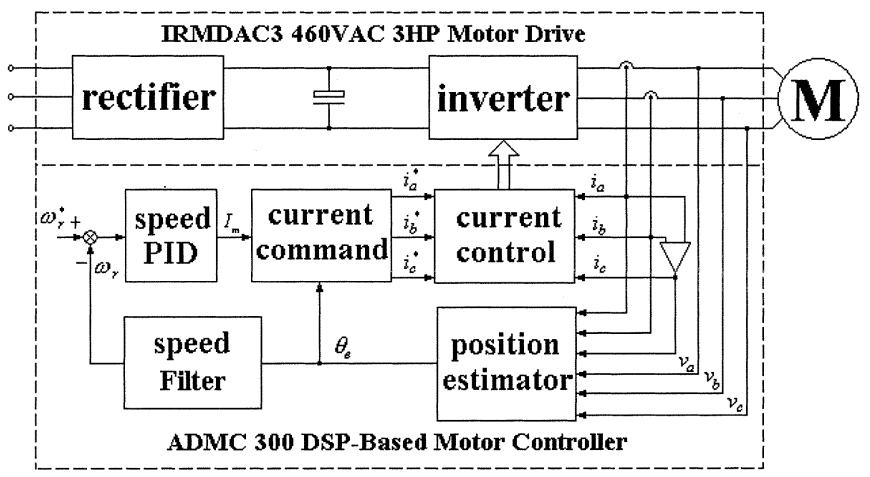

Fig. 5. Block diagram of the closed-loop position sensorless PM ac motor drive system.

measured in real-time. The third phase current that is required in the algorithm is calculated using the two measured phase currents.

For the nonsalient motor, if the current waveforms are in phase with the rotor position, the electromagnetic torque is proportional to the amplitude of the excitation current. Therefore, to generate the three-phase current commands using the estimated rotor position and the current amplitude, a look-up table for a sinusoidal function is established in the DSP as the current waveform command generator. A PI speed controller is implemented in the motor drive, which is considered as the torque command of the motor drive. Finally, the three-phase command currents are compared with the measured three-phase currents and the results are used as the duty cycle signals to generate the three-phase PWM signals for the switching power devices (IGBTs).

As known, in a digital motion control system, the duty cycle value can be updated once (or twice if the double update PWM mode is used) in each PWM cycle. In a conventional motor controller with a position sensor, there is only one interruption service routine, which has the same frequency as the PWM. In the position sensorless motor controller, however, the position sensorless algorithm requires high bandwidth voltage measurements. Therefore, two different interruption service routines are required.

In the method implemented, one of the routines is used to estimate the rotor position signals, and the second routine is used to employ the conventional motor control algorithms. This is because when three phases are excited with currents, the voltage of one phase has 6 possible values in each PWM cycle. Therefore, in order to sample the values of the phase voltages accurately, the A/D converter should have much higher sampling rate than the PWM frequency.

It should be emphasised here that the accuracy of the position estimation is directly proportional to the sampling frequency. Experiences indicate that the frequency of the interruption service routine should be at least 6 times the PWM frequency.

The highest possible sampling rate of the A/D converter used in this study is $31.5 \mathrm{kHz}$. Therefore, the frequency of PWM and the motor control interruption service routine of the position sensorless motor drive is selected as $5208 \mathrm{~Hz}$. So, the sampling rate of the A/D converter is 6 times higher than the PWM frequency. 


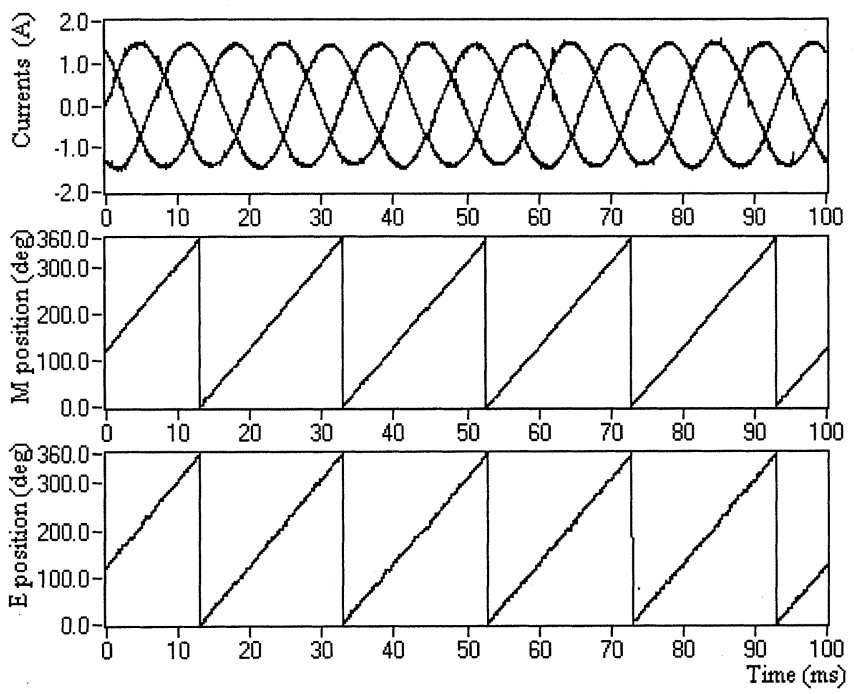

Fig. 6. The real-time experimental results obtained from the closed-loop motor drive operating at $107 \mathrm{rpm}$ without the shaft mounted position sensor: three-phase measured currents (top), measured position (middle) and estimated position (bottom)

Since the estimated rotor position values are used only once in the motor control interruption service routine per PWM cycle, the six estimated rotor position values within one PWM cycle are averaged in the real time system. Therefore, the estimation noises are reduced by this averaging method, and the closed-loop sensorless position estimator was implemented successfully.

\section{Starting Strategy in the Real Time Drive}

Since there is no rotor position data available at standstill, most of the position sensorless techniques presented in the literatures are not self-starting. Therefore, a special starting strategy should be accommodated in the controller until the position algorithm generates an accurate estimation of the rotor position.

In the position estimation method proposed in this paper, an initial value of the rotor position is required. Fortunately, the auto-correctional feature of the algorithm not only responds to the parameter variations and the measurement errors but also corrects the initial position value. For example, if the predetermined initial position is different than the actual rotor position, the phase difference between the initial rotor position and the phase angle of the flux linkage increments is fed back through the internal loop of the algorithm. Using the phase difference, the robust algorithm corrects the rotor position increment and updates the rotor position value, and after few sampling cycles, the estimated rotor position can converge to the actual value.

As will be demonstrated later, the proposed method can be used to start the PMAC motor from standstill in real time. This implies that the position estimation algorithm can predict the rotor position from a random initial value (such as zero). If the estimated rotor position is used as the position feedback in the controller, the motor may produce lower or even negative electromagnetic torque initially, which may affect the starting performance of the motor drive slightly. However, the estimated
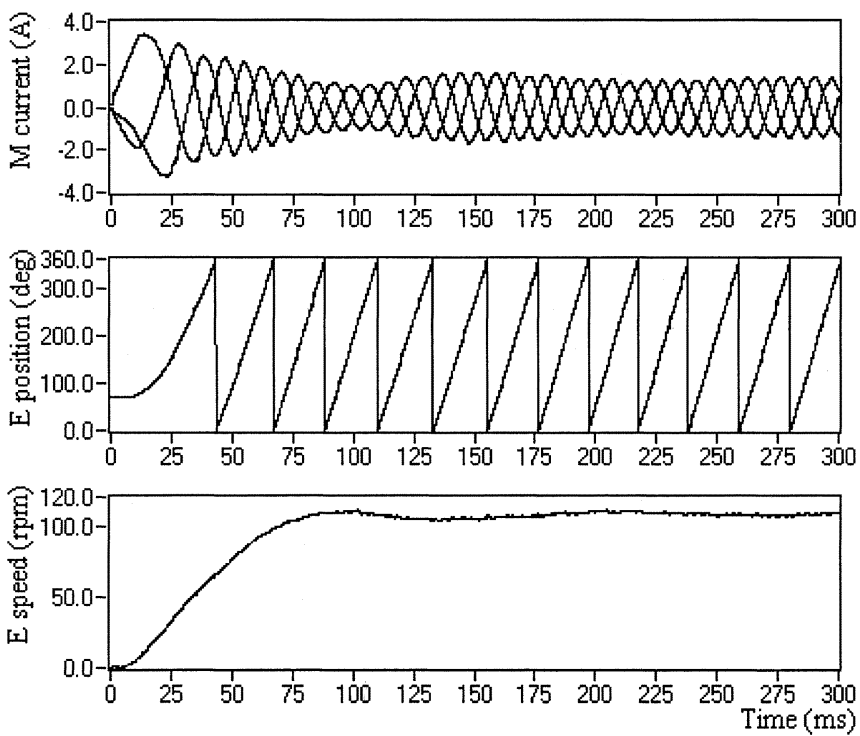

Fig. 7. Measured dynamic starting performance of the closed-loop position sensorless motor drive: Phase currents (top), the estimated rotor position (middle) and the estimated speed (bottom).

position can converge to the actual position within the first electrical cycle and can thus implement synchronous control from standstill.

In comparison with other open-loop starting strategies, it was demonstrated experimentally that this method has shorter starting time and a higher dynamic performance, and it was observed that the starting procedure is smooth.

\section{Real-Time Experimental Results (Without the Shaft Position Sensor)}

To demonstrate the effectiveness of the position estimation algorithm, a number of real time experiments have been performed at various operating conditions.

Fig. 6 provides a set of steady-state test results while the sensorless motor drive operates at a speed of $107 \mathrm{rpm}$ (about $50 \mathrm{~Hz}$ ). The figure illustrates the waveforms of the measured three-phase currents, the measured rotor position and the estimated rotor position of the motor. As can be seen in the position waveforms, there is a negligible discrepancy between the estimated and the measured position data, and the actual current waveforms are very close to the desired waveforms (sinusoidal) that are generated using the estimated rotor position data.

The real-time experimental results were also obtained from the closed-loop motor drive operating at $10.7 \mathrm{rpm}$ (about $5 \mathrm{~Hz}$ ). A very good agreement between the estimated and the measured rotor position is also found in this test. The position errors in these tests were less than $3 \%$. However, significant variations were observed on the actual phase currents of the real motor. The main reason for this is the small mechanical load of the real drive at the low speeds, which was difficult to rectify. A low inertia PM ac generator is used as a load that is attached to a three-phase resistive load bank.

Finally, a dynamic test was performed and the results are given in Fig. 7. In this experiment, the position sensorless motor 
drive was accelerated from standstill to $107 \mathrm{rpm}$. The results demonstrate that the position sensorless motor drive can start smoothly without the help of any special starting strategy, and a reasonable dynamic performance is achieved.

\section{CONCLUSION}

The paper has proposed a novel position sensorless technique suitable for a wide range of PM ac motors. Although, the principles of the method are developed and explained for the motors without rotor saliency, the method is also applicable to rotors with saliency. Here the position sensing is much easier as the variations of the inductance with position can be utilized.

The method described here is based on an incremental fluxlinkage algorithm, which can form an internal closed loop and uses the real-time voltages and current of the motor. In addition, with the help of the phase-locked loop algorithm, the internal closed loop can eliminate the problems associated with the integration drift, which exists in the previous methods. It was also demonstrated using the real data and some synthetic errors that the method has high robustness and reliability against parameter changes of the motor and various measurement errors.

The position estimation algorithm is simple and not mathematically intensive, which makes the real-time implementation possible. By using the DSP based real-time system, it was demonstrated that the algorithm developed here can start from standstill and can estimate the rotor position accurately under the practical operating conditions, including the static and dynamic performance. The starting from standstill requires an initial value that need not be correct. An incorrect starting position influences the starting dynamics only, and in the worst scenario it is corrected within one electrical cycle.

In comparison with previous position sensorless techniques, it can be concluded that this novel method has two major features. Firstly, the algorithm has high robustness with respect to the parameter variations and the measurement errors and hence is suitable for practical applications. Secondly, the auto-correctional ability of the method makes it applicable for starting and operation at a wider speed range, including slower speeds.

\section{APPENDIX}

The parameters of the experiment motor.

Back EMF constant, $k_{e}: 3.785 \mathrm{~V} / \mathrm{rad} / \mathrm{s}$.

Number of poles pair, $\mathrm{p}: 28$.

Winding resistance, $\mathrm{R}: 6.4 \Omega$.

Equivalent winding inductance, $\mathrm{L}: 32.8 \mathrm{Mh}$.

\section{REFERENCES}

[1] K. Iizuka and H. Uzuhashi et al., "Microcomputer control for sensorless brushless motor," IEEE Trans. Ind. Applicat., vol. 21, pp. 595-601, May/June 1985.

[2] J. C. Moreira, "Indirect sensing for rotor flux position of permanent magnet AC motors operation over a wide speed range," in Proc. IEEE-IAS Аnnu. Meeting, 1994, pp. 401-407.

[3] S. Ogasawara and H. Akagi, "An approach to position sensorless drive for brushless DC motor," IEEE Trans. Ind. Applicat., vol. 27, pp. 928-933, Sept./Oct. 1991.

[4] L. A. Jones and J. H. Lang, "A state observer for the permanent magnet synchronous motor," IEEE Trans. Ind. Electron., vol. 36, pp. 374-382, Aug. 1989.
[5] J. S. Kim and S. K. Sul, "High performance PMSM drives without rotational position sensors using reduced order observer," in Proc. Conf. IEEE IAS Annu. Meeting, 1995, pp. 75-82.

[6] M. Tomita and T. Senjyu et al., "New sensorless control for brushless DC motor using disturbance observers and adaptive velocity estimations," IEEE Trans. Ind. Electron., vol. 45, pp. 274-282, Apr. 1998.

[7] R. Dhaouadi, N. Mohan, and L. Norum, "Design and implementation of an extended Kalman filter for the state estimation of a permanent magnet synchronous motor," IEEE Trans. Power Electron., vol. 6, pp. 491-497, July 1991.

[8] S. Bolognani, R. Oboe, and M. Ziglitto, "Sensorless full-digital PMSM drive with EKF estimation of speed and rotor position," IEEE Trans. Ind. Electron., vol. 46, pp. 184-191, Feb. 1999.

[9] W. Rusong and G. R. Slemon, "A permanent magnet motor drive without a shaft sensor," in Proc. IEEE IAS Annu. Meeting, 1990, pp. 553-558.

[10] A. Consoli and S. Musumeci et al., "Sensorless vector and speed control of brushless motor drives," IEEE Trans. Ind. Electron., vol. 41, pp. 91-96, Feb. 1994.

[11] N. Ertugrul and P. P. Acarnley, "A new algorithm for sensorless operation of permanent magnet motors," IEEE Trans. Ind. Applicat., vol. 30, pp. 126-133, Jan./Feb. 1994

[12] A. B. Kulkarni and M. Ehsani, "A novel position sensor elimination technique for the interior permanent magnet synchronous motor drive," IEEE Trans. Ind. Applicat., vol. 28, pp. 144-150, Jan./Feb. 1992.

[13] S. Ogasawara and H. Akagi, "Implementation and position control performance of a position-sensorless IPM motor drive system based on magnetic saliency," IEEE Trans. Ind. Applicat., vol. 34, pp. 806-812, July/Aug. 1998.

[14] M. J. Corley and R. D. Lorenz, "Rotor position and velocity estimation for a salient-pole permanent magnet synchronous machine at standstill and high speeds," IEEE Trans. Ind. Applicat., vol. 34, pp. 784-789, July/Aug. 1998.

[15] A. H. Wijenayake, J. M. Bailey, and M. Naidu, "A DSP-based position sensor elimination method with an on-line parameter identification scheme for permanent magnet synchronous motor drives," in Proc. IEEE IAS Annu. Meeting, 1995, pp. 207-215.

[16] L. Ying and N. Ertugrul, "A novel position sensorless control for permanent magnet AC motors," in Proc. Int. Power Electron. Motion Control Conf. (IPEMC'00), Beijing, China, 2000, pp. 169-174.

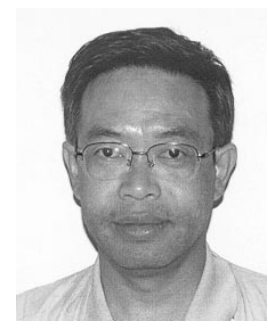

Li Ying received the B.Sc. and M.Sc. degrees in electrical and electronic engineering from the China University of Mining and Technology, China, in 1983 and 1986, respectively, and the Ph.D. degree from the University of Adelaide, Adelaide, Australia.

From 1987 to 1998, he was with Yunnan Polytechnic University, China. His research interests include real time control of motor drives, DSP-based applications, high efficiency and high performance motor drive design, and intelligent control systems in power electronics.

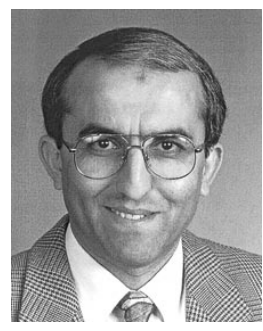

Nesimi Ertugrul (M'95) received the B.Sc. and M.Sc. degrees from the Istanbul Technical University, Istanbul, Turkey, in 1985 and 1989, respectively, and the Ph.D. degree from the University of Newcastle upon Tyne, U.K., in 1993.

$\mathrm{He}$ joined the University of Adelaide, Adelaide, Austrial, where since 1994, he has been a Senior Lecturer. He is the author of LabVIEW for Electric Circuits, Machines, Drives and Laboratories (Englewood Cliffs, NJ: Prentice-Hall, 2002). His research topics include sensorless operation and real time control of switched motor drives, fault tolerant motor drives, condition monitoring, and electric vehicles. His recent interests are macro machines and human machine interfaces.

Dr. Ertugrul serves on the Editorial Advisory Board for the International Journal of Engineering Education. 